\title{
Testing for Additive Outliers in Seasonally Integrated Time Series.
}

\author{
Niels Haldrup*, Antonio Montañés** and Andreu Sansó***
}

December 2004

\begin{abstract}
The detection of additive outliers in integrated variables has attracted some attention recently, see e.g. Shin et al. (1996), Vogelsang (1999) and Perron and Rodriguez (2003). This paper serves several purposes. We prove the inconsistency of the test proposed by Vogelsang, we extend the tests proposed by Shin et al. and Perron and Rodriguez to the seasonal case, and we consider alternative ways of computing their tests. We also study the effects of periodically varying variances on the previous tests and demonstrate that these can be seriously size distorted. Subsequently, some new tests that allow for periodic heteroskedasticity are proposed.
\end{abstract}

KEYWORDS: Additive outliers, outlier detection, integrated processes, periodic heteroscedasticity, seasonality.

JEL Classification: C12, C2, C22,

\footnotetext{
*Department of Economics, University of Aarhus, Building 322, DK-8000 Aarhus C, Denmark. E-mail: nhaldrup@econ.au.dk. ${ }^{* *}$ Department of Economic Analysis, University of Zaragoza, Gran Via 2, 50005 Zaragoza, Spain. E-mail: amontane@posta.unizar.es.***Department of Applied Economics University of The Balearic Islands, Ctra. Valldemossa km 7.5, 07122, Spain. E-mail: andreu.sanso@uib.es. The last author gratefully acknowledges the financial support from Conselleria d'Economia, Hisenda I Innovació del Govern Balear under PRIB-2004-10095.
} 


\section{Introduction}

Franses and Haldrup (1994), and Shin et al. (1996), for non-seasonal time series, and Haldrup et al. (2004), for seasonal data, have shown that the presence of additive outliers (AO) affects the limiting distribution of Dickey-Fuller type tests which tend to overreject the null in this case. The intuition behind these results is that the AOs introduce an MA-type autocorrelation component which distorts the size of the tests. As a consequence, it is necessary to check for the presence of outliers prior to testing for unit roots. With respect to the first step, i.e. the testing for the presence of outliers in $\mathrm{I}(1)$ variables, Shin et al. (1996) (SSL hereafter), Vogelsang (1999) and Perron and Rodriguez (2003), (PR hereafter), have proposed some new tests. Concerning the second aspect, i.e. the correction for the outliers when testing for unit roots, this has been considered by Franses and Haldrup (1994), Haldrup et al. (2004), Shin et al. (1996) and Vogelsang (1999). One of the suggestions of the last author is to use modified Phillips-Perron (1988) tests. Franses and Haldrup proposed to extend the auxiliary regression by including dummy variables to control for the AOs. whilst Shin et al. suggested to consider the observation affected by AOs as a missing observation and replace this by its expected value under the hypothesis of a unit root.

In this paper we first review the tests proposed by Vogelsang, Perron and Rodriguez, and Shin et al. We prove the inconsistency of the test proposed by the first author. Perron and Rodriguez (2003) have already shown that the iterative procedure based on this test is inconsistent, however, we demonstrate that the test itself is not consistent. Some simulation experiments show the poor performance that exists even in finite samples. Next, we extend the tests proposed by PR and SSL in several ways: we adapt the tests to the seasonal case and we consider different ways of computing the tests. By Monte Carlo experiments the performance of these alternative tests is studied.

Next, we study the effects of periodically varying variances on the PR and SSL tests. This feature of the seasonal integrated processes has been pointed out, by among others, Burridge and Wallis (1990), Burridge and Taylor (2001) and Franses (1996). We show that the tests for the detection of AOs in (seasonally) integrated processes have serious size distortions if the periodic heteroscedasticity is not taken into account, and hence we suggest some simple modifications of the tests that are robust to this feature.

\section{Testing for additive outliers in integrated time series}

Consider the univariate process generated by

$$
y_{t}=y_{t-s}+u_{t}
$$

where $u_{t}$ is an $\mathrm{I}(0)$ process and $s$ indicates the number of observations per year. 
The observed variable is

$$
z_{t}=\mu_{t}+y_{t}+\theta \delta_{t}
$$

where $\mu_{t}$ collects the deterministic terms and $\delta_{t}$ is a Bernouilli-type variable independent of $u_{t}$, such that $P\left(\delta_{t}=1\right)=P\left(\delta_{t}=-1\right)=p / 2, P\left(\delta_{t}=0\right)=1-p$, $0 \leq p<0.5$. Accordingly, $z_{t}$ is an integrated process subject to AOs. In the following we will consider some simple procedures to detect outliers in integrated processes.

\subsection{The Vogelsang test}

The test proposed by Vogelsang (1999) is based on estimating by OLS the sequence of (spurious) regressions

$$
z_{t}=F(t / T)^{\prime} \widehat{\beta}+\widehat{\theta} D\left(T_{a o}\right)_{t}+\widehat{u}_{t}
$$

for any $T_{a o}=1,2, \ldots, T$, where $F(t / T)$ is a vector of deterministic terms such as time trends and seasonal dummy variables. $D\left(T_{a o}\right)_{t}$ is a dummy variable that takes value 1 for $t=T_{a o}$ and 0 otherwise. The test statistic is given by $\tau=$ $\sup _{T_{a o}}\left|t_{\widehat{\theta}}\left(T_{a o}\right)\right|$ and the null hypothesis of $\theta=0$ is rejected if $\tau$ is greater than a given critical value. Under the null hypothesis of $\theta=0$ and the assumption that $\lambda=T_{a o} / T$ remains fixed as $T$ grows, the asymptotic distribution of the test is given by (see Vogelsang, 1999):

$$
\tau \Rightarrow \sup _{\lambda}\left|\frac{W^{*}(\lambda)}{\left(\int_{0}^{1} W^{*}(r)^{2} d r\right)^{1 / 2}}\right|
$$

where $\Rightarrow$ denotes weak convergence of the associated probability measures and $W^{*}(r)$ are the residuals from the projection of $W(r)$, a standard Wiener process, onto the space spanned by $F(r)$ on $(0,1)$. This asymptotic distribution is free of nuisance parameters and is invariant to the autocorrelation structure of $u_{t}$. The next proposition establishes the asymptotic distribution of the test under the alternative hypothesis. The proof is shown in the Appendix.

Proposition 1 Under the alternative hypothesis of $\theta \neq 0$, the asymptotic distribution of $\tau$ is given by (4).

Thus, the test statistic has the same asymptotic distribution both under the null and under the alternative and the power will equal the size even asymptotically; hence the test proposed by Vogelsang (1999) is inconsistent ${ }^{1}$. One intuition behind this result is that the presence of additive outliers introduces a MA component in I(1) processes, see e.g. Franses and Haldrup (1994), however, the asymptotic distribution of the test, given by (4), is invariant to serial

\footnotetext{
${ }^{1}$ Of course, the test will be consistent against a sequence of alternatives where the size of the outliers are allowed to increase with the sample size of a given speed. However, in practice we believe this class of models is of little interest.
} 
correlation. Another intuition behind the proposition is that an additive outlier will become negligible compared to the $\mathrm{I}(1)$ stochastic trend component as the sample size tends to infinity ${ }^{2}$.

Some Monte Carlo experiments confirm these findings. Table 1 shows the detection frequencies of the test when there is a fixed outlier in the middle of the sample of a random walk. Four sample sizes, $T=\{50,100,200,400\}$, and values of $\theta=\{0,5,10,15\}$ are considered. When $\theta=0$, no outliers are present and around $96 \%$ of the times the test gets the correct conclusion of absence of outliers using Vogelsangs critical values. For $\theta>0$, the test only detects the outlier for large values of $\theta$ and small sample sizes (say 50). When the sample size grows the performance of the test quickly deteriorates because the influence of the outlier is hidden in the total variation of the variable.

\section{[insert table 1 about here]}

Systematic AOs are considered in table 2. Four different probabilities of outliers, $p=\{0.01,0.025,0.05,0.1\}$, two sample sizes, $T=\{100,400\}$, and values of $\theta=\{5,15\}$ are considered. The results of the experiments confirm that the test only detects a small amount of the effective number of outliers. Only for large magnitudes of the outlier (say, $\theta=15$ ) the total number of detected outliers almost corresponds to the actual number of AOs. As well as for fixed outliers, the sample size deteriorates the ratio between detected outliers and effective outliers.

\section{[insert table 2 about here]}

Hence, we conclude that the test proposed by Vogelsang (1999) is generally inadequate for the detection of outliers.

\subsection{The Shin et al. test}

Here we extend to seasonal data the outlier detection test suggested by Shin et al. (1996). The test, which is the uniformly most powerful unbiased test for $\theta=0$, is given by,

$$
\tau_{\Delta z}^{S S L}=\sup _{T_{a o}}\left|t_{\Delta z}^{S S L}\left(T_{a o}\right)\right|
$$

where,

$$
t_{\Delta z}^{S S L}\left(T_{a o}\right)=\frac{\Delta z_{T_{a o}+1}-\Delta z_{T_{a o}}}{\sqrt{2} \widehat{\sigma}}
$$

for $T_{a o} \in\{2, \ldots, T-1\}, \widehat{\sigma}^{2}=(T-3)^{-1}\left[\left(\sum_{t=2}^{T} \Delta z_{t}^{2}\right)-\Delta z_{t^{\prime}}^{2}-\Delta z_{t^{\prime}+1}^{2}\right], \Delta z_{t}=$ $z_{t}-z_{t-1}$ and $t^{\prime}$ is the time point at which $\max \left\{\left|d_{t}\right|:\left|d_{t}\right|>\max \left\{\left|\Delta z_{t}\right|,\left|\Delta z_{t+1}\right|\right\}\right\}$ and $d_{t}=2^{-1 / 2}\left(\Delta z_{t+1}-\Delta z_{t}\right)$.

\footnotetext{
${ }^{2}$ One way of making the test asymptotically consistent is by letting the magnitude of the outlier grow with the sample size $T$ in a particular way. One might question, however, whether this is a satisfactory way of solving the inconsistency problem.
} 
The test can be easily extended to seasonal data, in which case special attention must be taken with respect to the outliers located at the beginning and at the end of the sample. This amounts to $2 s$ observations and for the usual sample sizes used in economics, this could be an important fraction of the available information. Let us suppose that a single outlier is located at $T_{a o} \leq s$, in which situation all the information about $\theta$ is contained in $\Delta_{s} z_{T_{a o}+s}=y_{T_{a o}+s}-y_{T_{a o}}-\theta=u_{T_{a o}+s}-\theta$. If the outlier is not located in the tails of the sample, all the information about $\theta$ is contained in $\Delta_{s} z_{T_{a o}+s}=u_{T_{a o}+s}-\theta$ and $\Delta_{s} z_{T_{a o}}=u_{T_{a o}}+\theta$, whereas if $T_{a o}>T-s$ all the information is contained in $\Delta_{s} z_{T_{a o}}=u_{T_{a o}}+\theta$. Hence, under the assumption that $u_{t} \sim$ iid $N\left(0, \sigma_{u}^{2}\right)$ and $\theta \neq 0$

$$
\begin{cases}\Delta_{s} z_{T_{a o}+s} \sim \operatorname{iid} N\left(-\theta, \sigma_{u}^{2}\right) & \text { for } T_{a o} \leq s \\ \Delta_{s} z_{T_{a o}+s}-\Delta_{s} z_{T_{a o}} \sim \text { iid } N\left(-2 \theta, 2 \sigma_{u}^{2}\right) & \text { for } s<T_{a o} \leq T-s \\ \Delta_{s} z_{T_{a o}} \sim \operatorname{iid} N\left(\theta, \sigma_{u}^{2}\right) & \text { for } T_{a o}>T-s\end{cases}
$$

and, thus, the test statistic for seasonal data is given by

$$
\tau_{\Delta_{s} z}^{S S L}=\sup _{T_{a o}}\left|t_{\Delta_{s} z}^{S S L}\left(T_{a o}\right)\right|
$$

where

$$
t_{\Delta_{s} z}^{S S L}\left(T_{a o}\right)= \begin{cases}\widehat{\sigma}^{-1} \Delta_{s} z_{T_{a o}+s} & \text { for } T_{a o} \leq s \\ 2^{-1 / 2} \widehat{\sigma}^{-1}\left(\Delta_{s} z_{T_{a o}+s}-\Delta_{s} z_{T_{a o}}\right) & \text { for } s<T_{a o} \leq T-s \\ \widehat{\sigma}^{-1} \Delta_{s} z_{T_{a o}} & \text { for } T_{a o}>T-s\end{cases}
$$

With respect to the deterministic terms, these can be dealt with by prior regression of $\Delta_{s} z_{t}$ on the deterministic terms and proceeding the analysis by using the residuals. The robust variance can be computed as

$\widehat{\sigma}^{2}= \begin{cases}(T-s-1-k)^{-1}\left[\left(\sum_{t=s+1}^{T} \Delta_{s} z_{t}^{2}\right)-\Delta_{s} z_{T_{a o}+s}^{2}\right] & \text { for } T_{a o} \leq s \\ (T-s-2-k)^{-1}\left[\left(\sum_{t=s+1}^{T} \Delta_{s} z_{t}^{2}\right)-\Delta_{s} z_{T_{a o}}^{2}-\Delta_{s} z_{T_{a o}+s}^{2}\right] & \text { for } s<T_{a o} \leq T-s \\ (T-s-1-k)^{-1}\left[\left(\sum_{t=s+1}^{T} \Delta_{s} z_{t}^{2}\right)-\Delta_{s} z_{T_{a o}}^{2}\right] & \text { for } T_{a o}>T-s\end{cases}$

where $k$ is the number of deterministic regressors. Other robust estimators of the variance can be used, such as the median absolute deviation (MAD) and the trimmed standard deviation.

Once an outlier is detected, Shin et al. suggest to treat this as a missing observation and replace it with its forecast under the null hypothesis of a random walk. That is, suppose that an AO was found at $T_{a o}$, then the contaminated observation $z_{T_{a o}}$ has to be replaced by: $\widehat{z}_{T_{a o}}=E\left(z_{T_{a o}} \mid z_{T_{a o}-1}, z_{T_{a o}-2}, \ldots\right)=$ $z_{T_{a o}-s}$. Then, the new series with the corrected observation must be checked for the presence of new outliers and the corresponding observations replaced by its forecast. The iterative procedure stops when no additional outlier is found. 


\subsection{The PR test}

The PR test (Perron and Rodriguez, 2003) is based on the auxiliary regression:

$$
\Delta z_{t}=\theta\left[D\left(T_{a o}\right)_{t}-D\left(T_{a o}\right)_{t-1}\right]+v_{t}
$$

where $D\left(T_{a o}\right)_{t-j}=1$ when $t=T_{a o}+j$ and 0 otherwise, so that, under the null hypothesis of $\theta=0$

$$
\begin{aligned}
\widehat{\theta}\left(T_{a o}\right) & = \begin{cases}\frac{1}{2}\left(\Delta z_{T_{a o}}-\Delta z_{T_{a o}+1}\right) & T_{a o} \leq T-1 \\
\Delta z_{T_{a o}} & T_{a o}=T\end{cases} \\
& = \begin{cases}\frac{1}{2}\left(u_{T_{a o}}-u_{T_{a o}+1}\right) & T_{a o} \leq T-1 \\
u_{T_{a o}} & T_{a o}=T\end{cases}
\end{aligned}
$$

Perron and Rodriguez (2003) propose to estimate the variance of $\widehat{\theta}$ as: $\operatorname{var}\left[\widehat{\theta}\left(T_{a o}\right)\right]_{P R}=$ $\frac{1}{2}(\widehat{R}(0)-\widehat{R}(1))$ where $\widehat{R}(j)=T^{-1} \sum_{t=s+j+1}^{T} \widehat{v}_{t} \widehat{v}_{t-j}$ and $\widehat{v}_{t}$ are the OLS residuals from (7). Defining $t^{P R}\left(T_{a o}\right)=\sqrt{2} \widehat{\theta}\left(T_{a o}\right)(\widehat{R}(0)-\widehat{R}(1))^{-1 / 2}$, the test statistic reads

$$
\tau^{P R}=\sup _{T_{a o}}\left|t^{P R}\left(T_{a o}\right)\right|
$$

Note however, that for $T_{a o}=T$ the $t$-statistic to be computed should be $t^{P R}\left(T_{a o}\right)=\widehat{\delta}\left(T_{a o}\right) \widehat{R}(0)^{-1 / 2}$.

The PR test can be easily extended to the seasonal case by considering the auxiliary regression

$$
\Delta_{s} z_{t}= \begin{cases}-\theta D\left(T_{a o}\right)_{t-s}+v_{t} & T_{a o} \leq s \\ \theta\left[D\left(T_{a o}\right)_{t}-D\left(T_{a o}\right)_{t-s}\right]+v_{t} & T_{a o}>s\end{cases}
$$

in which case,

$$
\widehat{\theta}\left(T_{a o}\right)= \begin{cases}-u_{T_{a o}+s} & T_{a o} \leq s \\ \frac{1}{2}\left(u_{T_{a o}}-u_{T_{a o}+s}\right) & s<T_{a o} \leq T-s \\ u_{T_{a o}} & T_{a o}>T-s\end{cases}
$$

and,

$$
\operatorname{var}\left[\widehat{\theta}\left(T_{a o}\right)\right]= \begin{cases}\widehat{R}(0) & T_{a o} \leq s \\ \frac{1}{2}(\widehat{R}(0)-\widehat{R}(s)) & s<T_{a o} \leq T-s \\ \widehat{R}(0) & T_{a o}>T-s\end{cases}
$$

In a similar way,

$$
t_{s}^{P R}\left(T_{a o}\right)= \begin{cases}\widehat{\theta}\left(T_{a o}\right) \widehat{R}(0)^{-1 / 2} & T_{a o} \leq s \\ \sqrt{2} \widehat{\theta}\left(T_{a o}\right)(\widehat{R}(0)-\widehat{R}(s))^{-1 / 2} & s<T_{a o} \leq T-s \\ \widehat{\theta}\left(T_{a o}\right) \widehat{R}(0)^{-1 / 2} & T_{a o}>T-s\end{cases}
$$


and the test statistic is given by

$$
\tau_{s}^{P R}=\sup _{T_{a o}}\left|t_{s}^{P R}\left(T_{a o}\right)\right| .
$$

If one outlier is located in the initial observations, $T_{a o} \leq s$, then $\Delta_{s} z_{T_{a o}+s}=$ $u_{T_{a o}+s}-\theta$. On the contrary, if the outlier lies in $s<T_{a o}^{\prime} \leq 2 s$, then $\Delta_{s} z_{T_{a o}^{\prime}}=$ $u_{T_{a o}^{\prime}}+\theta$ and $\Delta_{s} z_{T_{a o}^{\prime}+s}=u_{T_{a o}^{\prime}+s}-\theta$. Thus, one way to determine whether an outlier in the initial observations lies in $T_{a o} \leq s$ or in $T_{a o}+s$ could be to compare $\left|t_{s}^{P R}\left(T_{a o}+s\right)\right|=\left|\sqrt{2} \widehat{\theta}\left(T_{a o}+s\right)(\widehat{R}(0)-\widehat{R}(s))^{-1 / 2}\right|$ with $\left|t_{s}^{P R}\left(T_{a o}\right)\right|=$ $\left|\widehat{\theta}\left(T_{a o}\right) \widehat{R}(0)^{-1 / 2}\right|$. If $\left|t_{s}^{P R}\left(T_{a o}\right)\right|>\left|t_{s}^{P R}\left(T_{a o}+s\right)\right|$ the possible outlier lies in $T_{a o} \leq s$ or in $T_{a o}+s$ otherwise.

Concerning the deterministic terms, let us consider the auxiliary regression

$$
\Delta_{s} z_{t}=F(t / T)+\theta\left[D\left(T_{a o}\right)_{t}-D\left(T_{a o}\right)_{t-s}\right]+v_{t}
$$

where $F(t / T)$ is a vector of deterministic terms such as a constant, a trend, and seasonal dummy variables. OLS estimation of this equation is equivalent to

$$
\Delta_{s} z_{t}^{*}=\theta\left[D\left(T_{a o}\right)_{t}-D\left(T_{a o}\right)_{t-s}\right]^{*}+v_{t}
$$

where $\Delta_{s} z_{t}^{*}$ and $\left[D\left(T_{a o}\right)_{t}-D\left(T_{a o}\right)_{t-s}\right]^{*}$ are the residuals from the regression of $\Delta_{s} z_{t}$ and $\left[D\left(T_{a o}\right)_{t}-D\left(T_{a o}\right)_{t-s}\right]$ on $F(t / T)$, respectively. But note that for $F(t / T)$ being a constant or $F(t / T)=\sum_{q=1}^{s} D_{q t}$ being seasonal dummy variables, we have that, if $T_{a o}>s$, it holds that: $\left[D\left(T_{a o}\right)_{t}-D\left(T_{a o}\right)_{t-s}\right]^{*}=$ $\left[D\left(T_{a o}\right)_{t}-D\left(T_{a o}\right)_{t-s}\right]$. Then, we can use the auxiliary regression

$$
\Delta_{s} z_{t}^{*}=\theta\left[D\left(T_{a o}\right)_{t}-D\left(T_{a o}\right)_{t-s}\right]+v_{t}
$$

that is, to use the demeaned variable. Given that under our assumption $\Delta_{s} z_{t}$ is stationary, demeaning will not affect the critical values. Hence, it is enough to compute the critical values for the most simple regression.

Once an outlier has been detected, Perron and Rodriguez (2003) suggest to drop the corresponding observation. With seasonal data, this procedure cannot be followed given that it will distort the seasonal autocorrelation structure of the data. For instance, eliminating one observation in one quarter will mean that the corresponding year will have only three quarters. Hence, we suggest to follow the procedure suggested by Shin et al. (1996) and substitute the observation of the outlier by its forecast under the hypothesis of a seasonal random walk with deterministic components.

We have simulated the critical values associated with the test (10). It occurs that the fractiles are practically identical to those of Perron and Rodriquez (2003) where it is the total number of observations that matter for the relevant distribution. These findings apply regardless of the deterministics that have been conditioned upon in the construction of the test. 


\subsection{Monte Carlo Experiments}

In this subsection we study the finite sample performance of the above tests for detecting outliers in seasonal data, i.e. $\tau_{s}^{P R}$, and $\tau_{\Delta_{s} z}^{S S L}$. The Monte Carlo experiments are similar to those of Perron and Rodriguez (2003). The DGP is given by:

$$
\begin{aligned}
z_{t} & =\sum_{j=1}^{m} \delta_{j} D\left(T_{a o, j}\right)_{t}+y_{t} \\
\left(1-L^{4}\right)^{d} y_{t} & =v_{t} \\
v_{t} & =\rho v_{t-4}+\varepsilon_{t}+\theta \varepsilon_{t-4} \\
\varepsilon_{t} & \sim i i d N(0,1)
\end{aligned}
$$

In all experiments 3000 replications were used and $m=4$. Tables 3 to 6 show the size and power of the tests. To save space, for the SSL test only the results computing the variance with (6) are shown. Qualitatively similar results were obtained using the median absolute deviation and the trimmed standard deviation estimators.

The $\tau_{\Delta_{s} z}^{S S L}$ test has serious size problems for autocorrelated errors and for stationary processes. Moreover, the $\tau_{\Delta_{s} z}^{S S L}$ test seems the most powerful test but it outperforms the $\tau_{s}^{P R}$ test for the cases where its size distortion is bigger and in terms of size-adjusted powers the test seems less attractive. Overall, the $\tau_{s}^{P R}$ test seems to be the best test as it has the correct size in all the cases as well as good power. The rejection frequencies for the $\tau_{s}^{P R}$ test are almost identical to the ones reported by Perron and Rodriguez (2003) in their simulation experiments for the non-seasonal case.

\section{Periodic heteroscedasticity}

Let us suppose that the process is a seasonal random walk with periodic heteroscedasticity: $y_{t}=y_{t-s}+u_{t}$, with $u_{t} \sim i i d\left(0, \sigma_{[(t-1) / s] s}^{2}\right)$, where [.] is the operator taking the integer part of its argument. That is, each season follows a random walk with different variance in the innovations. This process has been considered by Burridge and Wallis (1990), Burridge and Taylor (2001) and Franses (1996) among others. In this context a representation of the process could be obtained by working with the annual process

$$
Y_{n}=Y_{n-1}+U_{n}
$$

where $n=[t / s]+1, Y_{n}=\left(y_{(n-1) s+1}, y_{(n-1) s+2}, \ldots, y_{(n-1) s+s-1}\right)^{\prime}$,

$U_{n}=\left(u_{(n-1) s+1}, \quad u_{(n-1) s+2}, \ldots, u_{(n-1) s+s-1}\right)^{\prime}$ and $E\left(U_{j} U_{j}^{\prime}\right)=\operatorname{diag}\left\{\sigma_{q}^{2}\right\}, q=$ $\{1,2, \ldots, s\}, j=1, \ldots,[T / s]$.

Table 7, Panel A shows the rejection frequencies of the Perron-Rodriguez test (8) when there is periodic heteroscedasticity. This DGP was also considered in 
Burridge and Taylor (2001). As it is observed, the periodic variances introduce a significant size distortion of the test. To avoid this poor performance, the PR test statistics could be modified according the periodic nature of the variances. Hence, we can define the statistic:

$$
\tau_{P H}^{P R}=\sup _{T_{a o}}\left|t_{P H}^{P R}\left(T_{a o}\right)\right|
$$

where

$$
t_{P H}^{P R}\left(T_{a o}\right)=\left\{\begin{array}{ll}
\widehat{\theta}\left(T_{a o}\right) \widehat{R}_{q}(0)^{-1 / 2} & T_{a o} \leq s \\
\sqrt{2} \widehat{\theta}\left(T_{a o}\right)\left(\widehat{R}_{q}(0)-\widehat{R}_{q}(1)\right)^{-1 / 2} & s<T_{a o} \leq T-s \\
\widehat{\theta}\left(T_{a o}\right) \widehat{R}_{q}(0)^{-1 / 2} & T_{a o}>T-s
\end{array},\right.
$$

$q=t-[(t-1) / s] \times s$ is the season, $\widehat{R}_{q}(j)=[T / s]^{-1} \sum_{n=j+1}^{[T / s]} \widehat{v}_{(n-1) s+q} \widehat{v}_{(n-1-j) s+q}$, and $\widehat{\theta}\left(T_{a o}\right)$ is the OLS estimate of $\theta$ in (7). That is, the variance and autocovariances are estimated using only the observations corresponding to the same season where the (possible) additive outlier is located.

Another strategy could be to pretest for periodic variances and, if periodicity is detected, then compute $\tau_{P H}^{P R}$, otherwise, compute $\tau_{s}^{P R}$. Let us denote as $\tau_{P-P H}^{P R}$ the supremum statistic computed with this procedure.

Table 7 Panel B shows that $\tau_{P H}^{P R}$ and $\tau_{P-P H}^{P R}$ have good size when the process is a random walk with periodic variances. Tables 8 to 11 show the size and power of these two tests for non-heteroscedastic processes. These results can be compared with those of Tables 3 to 6 . It seems that the new tests perform almost equally compared to $\tau_{s}^{P R}$ for these DGPs. Hence, we strongly recommend to use the tests that are robust to periodic heteroscedasticity.

\section{Empirical applications}

In order to illustrate the performance of the procedures for outlier detection, we have applied the tests to the analysis of US money demand. To that end, we have selected the most liquid definition of money demand, considering both the currency component of the US money stock, measured by M1, as well as the currency in circulation in the US economy. We will refer to these as CCM1 and $\mathrm{CC}$, respectively. The variables have been made real by using the US consumer price index as deflator. The monthly data covers the period 1947:1-2004:2 and the data are from the Board of Governors of the Federal Reserve System (see http://www.forecasts.org/data ).

Figures 1-3 display the variables and their first regular and first seasonal differences, respectively. These figures show that the variables exhibit similar behavior. Also, the series do not seem stationary, they exhibit a clear seasonal component and finally, they take values abnormally high in some periods. More precisely, we can relate these abnormal behaviour to the end of 1999 and the first half of 2001 episodes. Thus, it will be interestsing to see whether we can identify these as being outliers using the various tests. 
Table 12 reports the values of the $\tau_{P H}^{P R}, \tau_{s}^{P R}$ and $\tau_{\Delta_{s} z}^{S S L}$ tests. Formal F-tests for non-periodic heteroscedasticity could not be rejected and hence the results for $\tau_{P H}^{P R}$ and $\tau_{s}^{P R}$ are expected to be similar. For the currency component of the US money stock, CCM1, it can be seen that the $\tau_{P H}^{P R}$ and $\tau_{s}^{P R}$ tests implies the presence of 4 additive outliers (identically dated) whereas the $\tau_{\Delta_{s} z}^{S S L}$ test identifies one less outlier. Two of the outliers are clearly associated with the Y2K-effect: December 1999, and January 2000.

The results of the currency in circulation, $\mathrm{CC}$, are rather similar, although slight modifications exist. First, we observe that the $\tau_{P H}^{P R}$ statistic detects the existence of 6 outliers, the $\tau_{s}^{P R}$ tests identifies 5 outliers whilst $\tau_{\Delta_{s} z}^{S S L} \operatorname{detects} 4$ outliers. The tests generally agree about the four outliers from November 1999 through January 2000, i.e. an "extended" Y2K phenomenon. In fact, the $\tau_{P H}^{P R}$ test suggests the outlier episode to start in October 1999. The February 2002 outlier is common to all tests (as for CCM1) as are the June 2001 observation associated with the $\tau_{P H}^{P R}$ and $\tau_{s}^{P R}$ tests.

\section{Conclusions}

In this paper we have considered three exisiting tests for the detection of additive outliers in integrated processes and have extended these in several ways. First, we have shown that the test proposed by Vogelsang (1999) is inconsistent. Second, we have extended the procedures suggested by Shin et al. (1996) and Perron and Rodriguez (2003) to the seasonal case. Monte Carlo experiments have shown that the latter test outperforms the former in terms of size, which is almost equal to the nominal one in all the (homoscedastic) scenarios considered. Third, it has been reported that the presence of periodic heteroscedasticity introduces a size distortion in the previous tests. Consequently, two new tests that consider this feature are proposed and simulation experiments have shown their good performance in terms of both power and size.

\section{References}

[1] Burridge, P. and K.F. Wallis (1990). Seasonal adjustment and Kalman filtering: extension to periodic variances. Journal of Forecasting. 9, 109118.

[2] Burridge, P. and A.M.R. Taylor (2001). On regression based tests for seasonal unit roots in the presence of periodic heteroscedasticity. Journal of Econometrics. 104, 91-117.

[3] Franses, P. H. (1996). Periodicity and Stochastic Trends in Economic Time Series. Oxford University Press.

[4] Franses, P. H., and N. Haldrup, (1994). The effects of additive outliers on tests for unit roots and cointegration. Journal of Business and Economic Statistics. 12, 471-478. 
[5] Franses, P. H., and R. Paap (2004). Periodic Time Series Models. Oxford University Press.

[6] Haldrup, N., A. Montañés and A. Sansó (2004). Measurement errors and outliers in seasonal unit root testing. Forthcoming in Journal of Econometrics.

[7] Perron, P. and G. Rodriguez (2003): Searching for Additive Outliers in Nonstationay Time Series. Journal of Time Series Analysis. 24, 193-220.

[8] Phillips, P. C. B. and P. Perron, (1988). Testing for a unit root in time series regression. Biometrika 75, 335-346.

[9] Shin, D.W., S. Sarkar and J.H. Lee (1996). Unit root tests for time series with outliers. Statistics 83 Probability Letters. 30, 189-19\%.

[10] Vogelsang, T. J., (1999). Two simple procedures for testing for a unit root when there are additive outliers. Journal of Time Series Analysis. 20, 23752 .

\section{Appendix}

Proof. Proposition 1: From the expressions (1) and (2), define $S_{t}=\sum_{j} u_{j}$ and $\mu_{t}=F(t / T)^{\prime} \beta$, then the data-generation process under the alternative of $\theta \neq 0$ is given by $z_{t}=\mu_{t}+\left(S_{t}+\theta \delta_{t}\right)=\mu_{t}+S_{\eta t}$, whereas under the null $S_{\eta t}=S_{t}$. Let $D^{*}\left(T_{a o}\right)_{t}$ and $S_{\eta t}^{*}$ denote the residuals from the regression of $D\left(T_{a o}\right)_{t}$ and $z_{t}$ respectively on $F(t / T)$. The $t$-ratio testing $\theta=0$ can be written as (see Vogelsang, 1999, Appendix page 251):

$$
t_{\widehat{\theta}}\left(T_{a o}\right)=\frac{T^{-1 / 2} S_{\eta T a o}^{*}}{\left(T^{-2} \sum S_{\eta t}^{* 2}+o_{p}(1)\right)^{1 / 2}}
$$

Note that the presence of AOs does not modify neither the long-run variance of $\Delta y_{t}: \sigma^{2}=\lim _{T \rightarrow \infty} E\left(T^{-1} S_{\eta T}^{2}\right)=\lim _{T \rightarrow \infty} E\left(T^{-1}\left(S_{T}+\theta \delta_{T}\right)^{2}\right)=$ $\lim _{T \rightarrow \infty} E\left(T^{-1} S_{T}^{2}\right)$, nor the asymptotic limits of the numerator and the denominator: $T^{-1 / 2} S_{\eta[r T]}^{*} \Rightarrow \sigma W^{*}(r)$ and $T^{-2} \sum_{[r T]=1}^{T} S_{\eta[r T]}^{* 2} \Rightarrow \sigma^{2} \int_{0}^{1} W^{*}(r)^{2} d r$

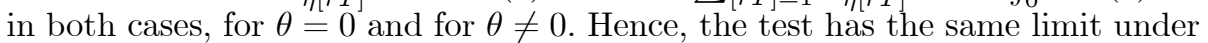
the null and the alternative hypothesis and therefore is inconsistent. 
Table 1: Detection frequencies for the Vogelsang $\tau$ statistic for a fixed outlier.

\begin{tabular}{ccccccc}
\hline \hline$\theta$ & $\mathrm{T}$ & $n_{a o}=0$ & \multicolumn{2}{c}{$n_{a o}=1$} & \multicolumn{2}{c}{$n_{a o}>1$} \\
\hline 0 & 50 & 0.971 & 0.012 & $(0)$ & 0.017 & $(0)$ \\
& 100 & 0.955 & 0.027 & $(0)$ & 0.018 & $(0)$ \\
& 200 & 0.962 & 0.018 & $(0)$ & 0.020 & $(0)$ \\
& 400 & 0.964 & 0.011 & $(0)$ & 0.025 & $(0)$ \\
5 & 50 & 0.781 & 0.202 & $(0.196)$ & 0.017 & $(0.012)$ \\
& 100 & 0.878 & 0.100 & $(0.085)$ & 0.022 & $(0.010)$ \\
& 200 & 0.948 & 0.031 & $(0.019)$ & 0.021 & $(0.001)$ \\
& 400 & 0.961 & 0.014 & $(0.003)$ & 0.025 & $(0.001)$ \\
10 & 50 & 0.299 & 0.672 & $(0.671)$ & 0.029 & $(0.029)$ \\
& 100 & 0.580 & 0.380 & $(0.378)$ & 0.040 & $(0.040)$ \\
& 200 & 0.791 & 0.179 & $(0.176)$ & 0.030 & $(0.017)$ \\
& 400 & 0.900 & 0.073 & $(0.065)$ & 0.027 & $(0.010)$ \\
15 & 50 & 0.080 & 0.890 & $(0.890)$ & 0.030 & $(0.030)$ \\
& 100 & 0.261 & 0.696 & $(0.696)$ & 0.043 & $(0.043)$ \\
& 200 & 0.490 & 0.478 & $(0.477)$ & 0.032 & $(0.029)$ \\
& 400 & 0.732 & 0.240 & $(0.232)$ & 0.028 & $(0.019)$ \\
\hline \hline
\end{tabular}

Notes: The data-generating process is given by $z_{t}=y_{t}+D(0.5 T)_{t}$, $t=1,2, \ldots T$, where $\Delta y_{t}=\varepsilon_{t}, \varepsilon_{t} \sim N(0,1)$. The auxiliary regression is given by: $y_{t}=\mu+\hat{\theta} D\left(T_{a o}\right)_{t}+\hat{u}_{t} .1000$ replications and $10 \%$ significance level were used. $n_{a o}$ stands for the number of outliers detected. The numbers in parenthesis are the frequencies of a correctly detected outlier in the middle of the sample. 
Table 2: Detection frequencies for the Vogelsang $\tau$ statistic for systematic outliers.

\begin{tabular}{cccccc}
\hline \hline$p$ & $\theta$ & $\mathrm{T}$ & $\overline{\bar{N}}_{a o}$ & $\bar{n}_{a o}$ & \\
\hline & 0 & 100 & 0 & 0.024 & $(0)$ \\
0.01 & & 400 & 0 & 0.235 & $(0)$ \\
& 5 & 100 & 1.023 & 0.142 & $(0.074)$ \\
& & 400 & 4.070 & 0.273 & $(0.038)$ \\
& 15 & 100 & 1.023 & 0.832 & $(0.756)$ \\
0.025 & & 400 & 4.070 & 1.238 & $(1.047)$ \\
& 5 & 100 & 2.509 & 0.245 & $(0.184)$ \\
& & 400 & 9.959 & 0.285 & $(0.079)$ \\
& 15 & 100 & 2.509 & 1.855 & $(1.794)$ \\
0.05 & & 400 & 9.959 & 2.519 & $(2.398)$ \\
& 5 & 100 & 5.074 & 0.338 & $(0.299)$ \\
& & 400 & 20.113 & 0.341 & $(0.163)$ \\
& 15 & 100 & 5.074 & 3.093 & $(3.073)$ \\
0.1 & & 400 & 20.113 & 4.265 & $(4.147)$ \\
& 5 & 100 & 9.974 & 0.394 & $(0.367)$ \\
& & 400 & 40.029 & 0.352 & $(0.205)$ \\
& 15 & 100 & 9.974 & 3.343 & $(3.333)$ \\
& & 400 & 40.029 & 4.585 & $(4.528)$ \\
\hline \hline
\end{tabular}

Notes: The data-generating process is given by $z_{t}=y_{t}+\theta \delta_{t}, t=$ $1,2, \ldots T$, where $\Delta y_{t}=\varepsilon_{t}, \varepsilon_{t} \sim N(0,1)$ and $\delta_{t}$ is an independent sequence of Bernouilli variables with $P\left(\delta_{t}=1\right)=P\left(\delta_{t}=-1\right)=p / 2$. The auxiliary regression is given by: $y_{t}=\mu+\hat{\theta} D\left(T_{a o}\right)_{t}+\hat{u}_{t} .1000$ replications and a $10 \%$ significance level were used. $\bar{N}_{a o}$ stands for the (average) number of outliers in the samples and $\bar{n}_{a o}$ for the (average) number of outliers detected. The numbers in parenthesis are the frequencies of correctly detected outliers. 
Table 3: Size of the PR test for non-stationary processes

\begin{tabular}{|c|c|c|c|c|c|c|c|c|c|c|}
\hline \multirow[b]{3}{*}{$\theta$} & \multicolumn{10}{|c|}{$\overline{\text { PANEL A: } d=1 ; \delta_{j}=0 \forall j ; \rho=0}$} \\
\hline & \multicolumn{5}{|c|}{$\tau_{s}^{P R}$} & \multicolumn{5}{|c|}{$\tau_{\Delta_{s} z}^{S S L}$} \\
\hline & $n_{1}$ & $n_{2}$ & $n_{3}$ & $n_{4}$ & $n_{>4}$ & $n_{1}$ & $n_{2}$ & $n_{3}$ & $n_{4}$ & $n_{>4}$ \\
\hline-0.8 & 0.047 & 0.002 & 0.000 & 0.000 & 0.000 & 0.299 & 0.064 & 0.011 & 0.000 & 0.000 \\
\hline-0.4 & 0.050 & 0.004 & 0.000 & 0.000 & 0.000 & 0.201 & 0.027 & 0.003 & 0.000 & 0.000 \\
\hline 0 & 0.054 & 0.003 & 0.000 & 0.000 & 0.000 & 0.053 & 0.003 & 0.001 & 0.000 & 0.000 \\
\hline 0.4 & 0.035 & 0.003 & 0.001 & 0.000 & 0.000 & 0.009 & 0.000 & 0.000 & 0.000 & 0.000 \\
\hline 0.8 & 0.020 & 0.004 & 0.001 & 0.001 & 0.001 & 0.005 & 0.000 & 0.000 & 0.000 & 0.000 \\
\hline$\rho$ & \multicolumn{10}{|c|}{ PANEL B: $d=1 ; \delta_{j}=0 \forall j ; \theta=0$} \\
\hline-0.8 & 0.031 & 0.004 & 0.000 & 0.000 & 0.000 & 0.383 & 0.141 & 0.052 & 0.020 & 0.007 \\
\hline-0.4 & 0.058 & 0.002 & 0.000 & 0.000 & 0.000 & 0.230 & 0.041 & 0.006 & 0.001 & 0.000 \\
\hline 0.4 & 0.030 & 0.004 & 0.001 & 0.001 & 0.001 & 0.003 & 0.000 & 0.000 & 0.000 & 0.000 \\
\hline 0.8 & 0.031 & 0.010 & 0.009 & 0.009 & 0.008 & 0.000 & 0.000 & 0.000 & 0.000 & 0.000 \\
\hline
\end{tabular}

Notes: DGP: $z_{t}=\sum_{j=1}^{m} \delta_{j} D\left(T_{a o, j}\right)_{t}+y_{t},\left(1-L^{4}\right)^{d} y_{t}=v_{t}, v_{t}=$ $\rho v_{t-4}+\varepsilon_{t}+\theta \varepsilon_{t-4}, \varepsilon_{t} \sim \operatorname{iidN}(0,1) .3 .000$ replications. $T=120.5 \%$ critical values. $n_{i}$ stands for the frequency of detecting the $i-t h$ outlier.

Table 4: Size of the PR test for stationary processes

\begin{tabular}{|c|c|c|c|c|c|c|c|c|c|c|}
\hline \multirow[b]{3}{*}{$\rho$} & \multicolumn{10}{|c|}{$\bar{c} d=0 ; \delta_{j}=0 \forall j ; \theta=0$} \\
\hline & \multicolumn{5}{|c|}{$\tau_{s}^{P R}$} & \multicolumn{5}{|c|}{$\tau_{\Delta_{s} z}^{S S L}$} \\
\hline & $n_{1}$ & $n_{2}$ & $n_{3}$ & $n_{4}$ & $n_{>4}$ & $n_{1}$ & $n_{2}$ & $n_{3}$ & $n_{4}$ & $n_{>4}$ \\
\hline 0.9 & 0.053 & 0.003 & 0.000 & 0.000 & 0.000 & 0.056 & 0.003 & 0.000 & 0.000 & 0.000 \\
\hline 0.7 & 0.051 & 0.002 & 0.000 & 0.000 & 0.000 & 0.092 & 0.005 & 0.001 & 0.000 & 0.000 \\
\hline 0.5 & 0.056 & 0.002 & 0.000 & 0.000 & 0.000 & 0.144 & 0.016 & 0.001 & 0.000 & 0.000 \\
\hline 0.3 & 0.055 & 0.001 & 0.000 & 0.000 & 0.000 & 0.206 & 0.034 & 0.005 & 0.000 & 0.000 \\
\hline 0 & 0.053 & 0.002 & 0.000 & 0.000 & 0.000 & 0.308 & 0.074 & 0.018 & 0.004 & 0.001 \\
\hline
\end{tabular}


Table 5: Power of the PR test for non-stationary processes

\begin{tabular}{|c|c|c|c|c|c|c|c|c|c|c|}
\hline \multirow[b]{3}{*}{$\theta$} & \multicolumn{10}{|c|}{$\overline{\text { PANEL A: } d=1 ; \delta_{j}=\{5,3,2,2\} \text { for } T_{a o}=\{30,55,77,100\} ; \rho=0}$} \\
\hline & \multicolumn{5}{|c|}{$\tau_{s}^{P R}$} & \multicolumn{5}{|c|}{$\tau_{\Delta_{s} z}^{S S L}$} \\
\hline & $n_{1}$ & $n_{2}$ & $n_{3}$ & $n_{4}$ & $n_{>4}$ & $n_{1}$ & $n_{2}$ & $n_{3}$ & $n_{4}$ & $n_{>4}$ \\
\hline-0.8 & 0.766 & 0.191 & 0.026 & 0.002 & 0.000 & 0.945 & 0.556 & 0.204 & 0.059 & 0.011 \\
\hline-0.4 & 0.945 & 0.410 & 0.077 & 0.006 & 0.000 & 0.993 & 0.700 & 0.268 & 0.078 & 0.015 \\
\hline 0 & 0.998 & 0.679 & 0.219 & 0.043 & 0.003 & 0.998 & 0.755 & 0.278 & 0.045 & 0.004 \\
\hline 0.4 & 1.000 & 0.827 & 0.370 & 0.100 & 0.016 & 1.000 & 0.621 & 0.133 & 0.016 & 0.001 \\
\hline 0.8 & 0.999 & 0.769 & 0.309 & 0.088 & 0.041 & 0.987 & 0.323 & 0.027 & 0.002 & 0.000 \\
\hline$\rho$ & \multicolumn{10}{|c|}{ PANEL B: $d=1 ; \delta_{j}=\{5,3,2,2\}$ for $T_{a o}=\{30,55,77,100\} ; \theta=0$} \\
\hline-0.8 & 0.370 & 0.051 & 0.002 & 0.000 & 0.000 & 0.811 & 0.429 & 0.173 & 0.063 & 0.021 \\
\hline-0.4 & 0.932 & 0.380 & 0.075 & 0.007 & 0.000 & 0.991 & 0.695 & 0.288 & 0.085 & 0.015 \\
\hline 0.4 & 1.000 & 0.867 & 0.445 & 0.134 & 0.029 & 1.000 & 0.641 & 0.125 & 0.013 & 0.001 \\
\hline 0.8 & 1.000 & 0.927 & 0.618 & 0.412 & 0.340 & 0.945 & 0.119 & 0.004 & 0.000 & 0.000 \\
\hline
\end{tabular}

Table 6: Power of the PR test for stationary processes

\begin{tabular}{|c|c|c|c|c|c|c|c|c|c|c|}
\hline \multirow[b]{3}{*}{$\rho$} & \multicolumn{10}{|c|}{$\bar{c} d=0 ; \delta_{j}=\{5,3,2,2\}$ for $T_{a o}=\{30,55,77,100\} ; \theta=0$} \\
\hline & \multicolumn{5}{|c|}{$\tau_{s}^{P R}$} & \multicolumn{5}{|c|}{$\tau_{\Delta_{s} z}^{S S L}$} \\
\hline & $n_{1}$ & $n_{2}$ & $n_{3}$ & $n_{4}$ & $n_{>4}$ & $n_{1}$ & $n_{2}$ & $n_{3}$ & $n_{4}$ & $n_{>4}$ \\
\hline 0.9 & 0.996 & 0.637 & 0.179 & 0.030 & 0.002 & 0.998 & 0.718 & 0.259 & 0.049 & 0.004 \\
\hline 0.7 & 0.975 & 0.502 & 0.117 & 0.014 & 0.001 & 0.995 & 0.651 & 0.208 & 0.040 & 0.005 \\
\hline 0.5 & 0.941 & 0.373 & 0.064 & 0.007 & 0.000 & 0.979 & 0.601 & 0.184 & 0.035 & 0.005 \\
\hline 0.3 & 0.844 & 0.248 & 0.038 & 0.004 & 0.000 & 0.957 & 0.520 & 0.168 & 0.037 & 0.007 \\
\hline 0 & 0.619 & 0.117 & 0.009 & 0.001 & 0.000 & 0.881 & 0.444 & 0.164 & 0.048 & 0.011 \\
\hline
\end{tabular}


Table 7: Size of thePR and SSL tests. Periodic heteroskedastic random walks

\begin{tabular}{|c|c|c|c|c|c|c|c|c|c|c|}
\hline & \multicolumn{10}{|c|}{ 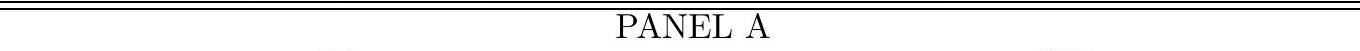 } \\
\hline \multirow[b]{2}{*}{$\sigma_{a}^{2}$} & \multicolumn{5}{|c|}{$\tau_{s}^{P R}$} & \multicolumn{5}{|c|}{$\tau_{\Delta_{s} z}^{S S L}$} \\
\hline & $\overline{n_{1}}$ & $\overline{n_{2}}$ & $n_{3}$ & $n_{4}$ & $n_{>4}$ & $n_{1}$ & $n_{2}$ & $n_{3}$ & $n_{4}$ & \\
\hline$\{3,1,3,1\}$ & 0.2130 & 0.0363 & 0.0037 & 0.0003 & 0.0003 & 0.2040 & 0.0347 & 0.0040 & 0.0003 & 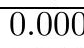 \\
\hline$\{30,1,30,1\}$ & 0.5703 & 0.2617 & 0.1053 & 0.0433 & 0.0150 & 0.5420 & 0.2280 & 0.0897 & 0.0370 & \\
\hline$\{3,1,1,1\}$ & 0.3087 & 0.0637 & 0.0140 & 0.0023 & 0.0000 & 0.3090 & 0.0583 & 0.0137 & 0.0013 & \\
\hline$\{30,1,1,1\}$ & 0.9683 & 0.873 & 0.7063 & 0.5347 & 0.3673 & 0.9327 & 0.7757 & 0.5863 & 0.4100 & 0.2 \\
\hline \multirow[t]{3}{*}{$3,3,1,1$} & 0.2253 & 0.0363 & 0.0050 & 0.0013 & 0.0003 & 0.2217 & 0.0313 & 0.0047 & 0.0013 & 0.0 \\
\hline & \multicolumn{10}{|c|}{ PANEL B } \\
\hline & \multicolumn{5}{|c|}{$\tau_{P H}^{P R}$} & \multicolumn{5}{|c|}{$\tau_{P-P H}^{P R}$} \\
\hline$\{1,1,1,1\}$ & 0.044 & 0.001 & 0.000 & 0.000 & 0.000 & 0.045 & 0.002 & 0.000 & 0.000 & \\
\hline$\{3,1,3,1\}$ & 0.053 & 0.001 & 0.000 & 0.000 & 0.000 & 0.058 & 0.002 & 0.000 & 0.000 & $0 .($ \\
\hline$\{30,1,30,1\}$ & 0.049 & 0.001 & 0.000 & 0.000 & 0.000 & 0.049 & 0.001 & 0.000 & 0.000 & 0.0 \\
\hline$\{3,1,1,1\}$ & 0.047 & 0.001 & 0.000 & 0.000 & 0.000 & 0.054 & 0.001 & 0.000 & 0.000 & 0.00 \\
\hline$\{30,1,1,1\}$ & 0.053 & 0.001 & 0.000 & 0.000 & 0.000 & 0.053 & 0.001 & 0.000 & 0.000 & 0.00 \\
\hline$\{3,3,1,1\}$ & 0.048 & 0.001 & 0.000 & 0.000 & 0.000 & 0.066 & 0.003 & 0.000 & 0.000 & 0.00 \\
\hline
\end{tabular}

Notes: DGP: $y_{t}=y_{t-4}+u_{t}, u_{t} \sim \operatorname{iidN}\left(0, \sigma_{t-[t / s] * s}^{2}\right) .3000$ replications.

$T=120.5 \%$ critical values are used. $n_{i}$ stands for the frequency of

detecting the $i-t h$ outlier.

Table 8: Size of the PR test robust to periodic heteroskedasticity. Nonstationary ARIMA processes

\begin{tabular}{|c|c|c|c|c|c|c|c|c|c|c|}
\hline \multirow[b]{3}{*}{$\theta$} & \multicolumn{10}{|c|}{ 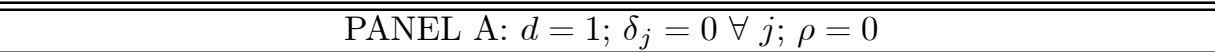 } \\
\hline & \multicolumn{5}{|c|}{$\tau_{P H}^{P R}$} & \multicolumn{5}{|c|}{$\tau_{P-P H}^{P R}$} \\
\hline & $n_{1}$ & $n_{2}$ & $n_{3}$ & $n_{4}$ & $n_{>4}$ & $n_{1}$ & $n_{2}$ & $n_{3}$ & $n_{4}$ & $n_{>4}$ \\
\hline-0.8 & 0.078 & 0.002 & 0.000 & 0.000 & 0.000 & 0.050 & 0.002 & 0.000 & 0.000 & 0.000 \\
\hline-0.4 & 0.064 & 0.002 & 0.000 & 0.000 & 0.000 & 0.046 & 0.002 & 0.000 & 0.000 & 0.000 \\
\hline 0.4 & 0.022 & 0.001 & 0.000 & 0.000 & 0.000 & 0.041 & 0.002 & 0.000 & 0.000 & 0.000 \\
\hline 0.8 & 0.004 & 0.000 & 0.000 & 0.000 & 0.000 & 0.019 & 0.002 & 0.000 & 0.000 & 0.000 \\
\hline$\rho$ & \multicolumn{10}{|c|}{ PANEL B: $d=1 ; \delta_{j}=0 \forall j ; \theta=0$} \\
\hline-0.8 & 0.030 & 0.000 & 0.000 & 0.000 & 0.000 & 0.025 & 0.000 & 0.000 & 0.000 & 0.000 \\
\hline-0.4 & 0.076 & 0.003 & 0.000 & 0.000 & 0.000 & 0.055 & 0.003 & 0.000 & 0.000 & 0.000 \\
\hline 0.4 & 0.023 & 0.001 & 0.000 & 0.000 & 0.000 & 0.037 & 0.001 & 0.000 & 0.000 & 0.000 \\
\hline 0.8 & 0.003 & 0.000 & 0.000 & 0.000 & 0.000 & 0.025 & 0.001 & 0.000 & 0.000 & 0.000 \\
\hline
\end{tabular}


Table 9: Size of the PR test robust to periodic heteroskedasticity. Stationary ARIMA processes

\begin{tabular}{|c|c|c|c|c|c|c|c|c|c|c|}
\hline \multirow[b]{3}{*}{$\rho$} & \multicolumn{10}{|c|}{$d=0 ; \delta_{j}=0 \forall j ; \theta=0$} \\
\hline & \multicolumn{5}{|c|}{$\tau_{P H}^{P R}$} & \multicolumn{5}{|c|}{$\tau_{P-P H}^{P R}$} \\
\hline & $n_{1}$ & $n_{2}$ & $n_{3}$ & $n_{4}$ & $n_{>4}$ & $n_{1}$ & $n_{2}$ & $n_{3}$ & $n_{4}$ & $n_{>4}$ \\
\hline 0.9 & 0.050 & 0.001 & 0.000 & 0.000 & 0.000 & 0.051 & 0.001 & 0.000 & 0.000 & 0.000 \\
\hline 0.7 & 0.047 & 0.001 & 0.000 & 0.000 & 0.000 & 0.046 & 0.003 & 0.000 & 0.000 & 0.000 \\
\hline 0.5 & 0.056 & 0.003 & 0.000 & 0.000 & 0.000 & 0.047 & 0.003 & 0.000 & 0.000 & 0.000 \\
\hline 0.3 & 0.061 & 0.003 & 0.000 & 0.000 & 0.000 & 0.056 & 0.004 & 0.000 & 0.000 & 0.000 \\
\hline 0 & 0.078 & 0.002 & 0.000 & 0.000 & 0.000 & 0.055 & 0.002 & 0.000 & 0.000 & 0.000 \\
\hline
\end{tabular}

Table 10: Power of the PR test robust to periodic heteroskedasticity. Nonstationary ARIMA processes

\begin{tabular}{|c|c|c|c|c|c|c|c|c|c|c|}
\hline \multirow[b]{3}{*}{$\theta$} & \multicolumn{10}{|c|}{ 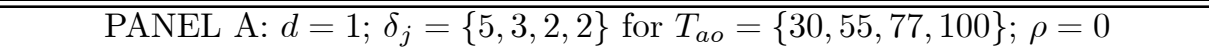 } \\
\hline & \multicolumn{5}{|c|}{$\tau_{P H}^{P R}$} & \multicolumn{5}{|c|}{$\tau_{P-P H}^{P R}$} \\
\hline & $n_{1}$ & $n_{2}$ & $n_{3}$ & $n_{4}$ & $n_{>4}$ & $n_{1}$ & $n_{2}$ & $n_{3}$ & $n_{4}$ & $n_{>4}$ \\
\hline-0.8 & 0.716 & 0.156 & 0.017 & 0.002 & 0.000 & 0.732 & 0.188 & 0.024 & 0.002 & 0.000 \\
\hline-0.4 & 0.936 & 0.379 & 0.059 & 0.006 & 0.000 & 0.947 & 0.426 & 0.084 & 0.013 & 0.000 \\
\hline 0 & 0.997 & 0.662 & 0.161 & 0.014 & 0.001 & 0.999 & 0.697 & 0.240 & 0.040 & 0.004 \\
\hline 0.4 & 1.000 & 0.823 & 0.275 & 0.039 & 0.003 & 1.000 & 0.839 & 0.368 & 0.095 & 0.009 \\
\hline 0.8 & 0.999 & 0.740 & 0.218 & 0.026 & 0.002 & 0.998 & 0.775 & 0.282 & 0.068 & 0.010 \\
\hline$\rho$ & \multicolumn{10}{|c|}{ PANEL B: $d=1 ; \delta_{j}=\{5,3,2,2\}$} \\
\hline-0.8 & 0.428 & 0.049 & 0.003 & 0.000 & 0.000 & 0.363 & 0.048 & 0.004 & 0.000 & 0.000 \\
\hline-0.4 & 0.920 & 0.340 & 0.048 & 0.004 & 0.000 & 0.930 & 0.367 & 0.075 & 0.007 & 0.000 \\
\hline 0.4 & 1.000 & 0.864 & 0.335 & 0.050 & 0.001 & 1.000 & 0.875 & 0.429 & 0.119 & 0.007 \\
\hline 0.8 & 1.000 & 0.883 & 0.374 & 0.063 & 0.002 & 1.000 & 0.878 & 0.408 & 0.097 & 0.005 \\
\hline
\end{tabular}

Table 11: Power of the PR test robust to periodic heteroskedasticity. Stationary ARIMA processes

\begin{tabular}{|c|c|c|c|c|c|c|c|c|c|c|}
\hline \multirow[b]{3}{*}{$\rho$} & \multicolumn{10}{|c|}{$\bar{c} d=0 ; \delta_{j}=\{5,3,2,2\}$ for $T_{a o}=\{30,55,77,100\} ; \theta=0$} \\
\hline & \multicolumn{5}{|c|}{$\tau_{P H}^{P R}$} & \multicolumn{5}{|c|}{$\tau_{P-P H}^{P R}$} \\
\hline & $n_{1}$ & $n_{2}$ & $n_{3}$ & $n_{4}$ & $n_{>4}$ & $n_{1}$ & $n_{2}$ & $n_{3}$ & $n_{4}$ & $n_{>4}$ \\
\hline 0.9 & 0.994 & 0.614 & 0.126 & 0.009 & 0.000 & 0.993 & 0.656 & 0.204 & 0.035 & 0.002 \\
\hline 0.7 & 0.973 & 0.451 & 0.076 & 0.007 & 0.000 & 0.973 & 0.488 & 0.109 & 0.017 & 0.001 \\
\hline 0.5 & 0.922 & 0.307 & 0.039 & 0.002 & 0.000 & 0.930 & 0.354 & 0.056 & 0.005 & 0.001 \\
\hline 0.3 & 0.807 & 0.209 & 0.023 & 0.001 & 0.000 & 0.827 & 0.256 & 0.038 & 0.004 & 0.000 \\
\hline 0 & 0.595 & 0.108 & 0.009 & 0.001 & 0.000 & 0.613 & 0.123 & 0.015 & 0.001 & 0.000 \\
\hline
\end{tabular}


Table 12: Detected outliers for US M1, CCM1, and the US currency in circulation, CC.

\begin{tabular}{ccccccc}
\hline \hline & \multicolumn{2}{c}{$\tau_{P H}^{P R}$} & \multicolumn{2}{c}{$\tau_{s}^{P R}$} & \multicolumn{2}{c}{$\tau_{\Delta_{s} z}^{S S L}$} \\
\hline \multirow{4}{*}{ CCM1 } & 7.17 & $1999: 12$ & 5.43 & $1999: 12$ & 4.55 & $1999: 12$ \\
& 7.08 & $2000: 1$ & 6.19 & $2000: 1$ & 5.31 & $2000: 1$ \\
& 5.89 & $2001: 2$ & 4.91 & $2001: 2$ & 4.02 & $2001: 2$ \\
& 5.11 & $2001: 6$ & 4.57 & $2001: 6$ & & \\
\hline \multirow{6}{*}{ CC } & 6.12 & $1999: 10$ & & & & \\
& 9.77 & $1999: 11$ & 6.93 & $1999: 11$ & 5.83 & $1999: 11$ \\
& 16.03 & $1999: 12$ & 10.26 & $1999: 12$ & 9.33 & $1999: 12$ \\
& 10.59 & $2000: 1$ & 8.77 & $2000: 1$ & 7.61 & $2000: 1$ \\
& 5.85 & $2001: 2$ & 4.97 & $2001: 2$ & 4.09 & $2001: 2$ \\
\hline \hline
\end{tabular}

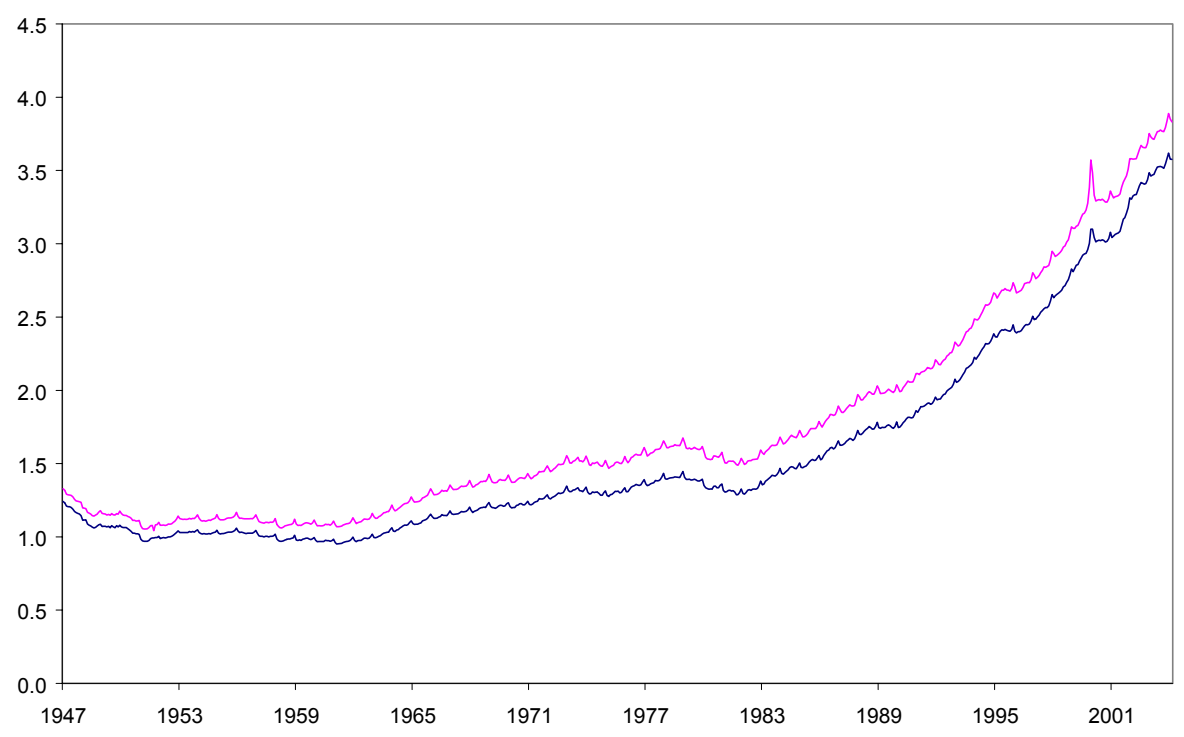

Figure 1: M1 and Currency in Circulation in the USA. Monthly data.: 19472003. 




Figure 2: M1 and Currency in Circulation in the USA. Monthly data.: 19472003. First differences.

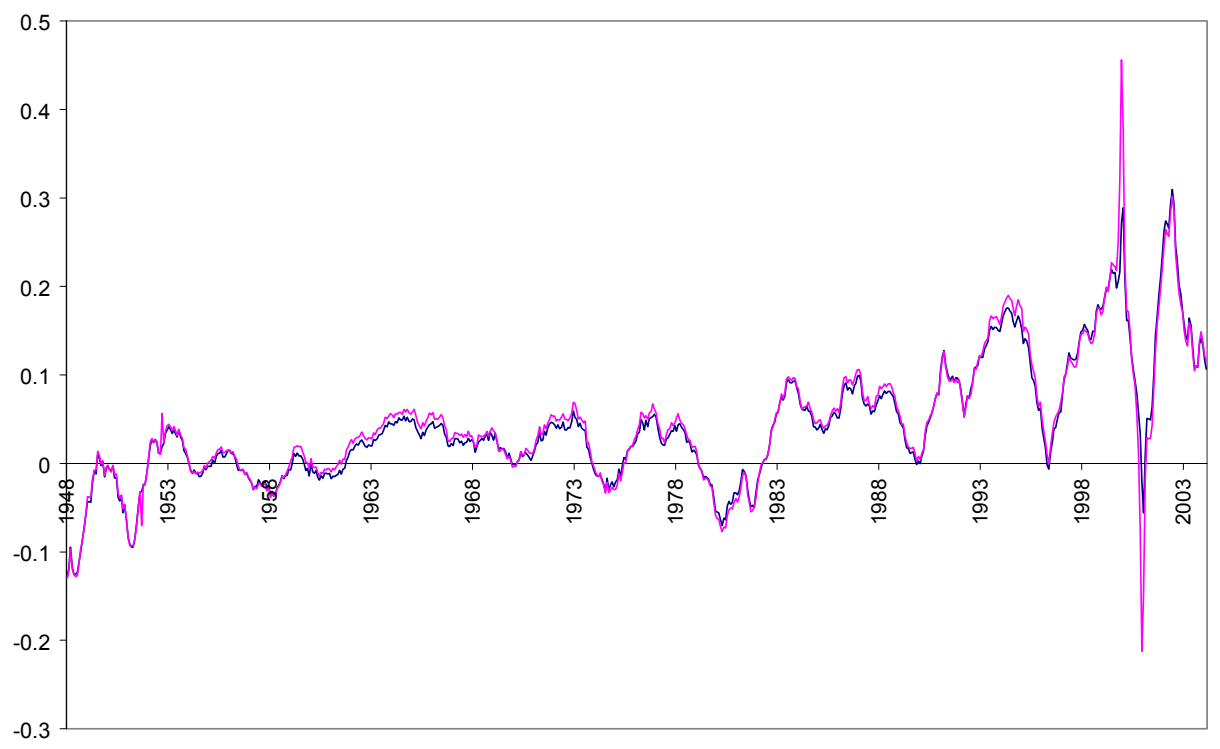

Figure 3: M1 and Currency in Circulation in the USA. Monthly data.: 19472003. Seasonal differences. 